\title{
Labyrinthe
}

29 | 2008 (1)

Ce que le libéralisme promet

\section{Nozick, le liberatisme et l'utopie des dix robinsons}

\section{Michel Baudouin}

\section{(2) OpenEdition}

\section{Journals}

\section{Édition électronique}

URL : http://journals.openedition.org/labyrinthe/3433

DOI : $10.4000 /$ labyrinthe.3433

ISSN : 1950-6031

\section{Éditeur}

Hermann

\section{Édition imprimée}

Date de publication : 11 janvier 2008

Pagination : 41-50

ISBN : 9782952613163

\section{Référence électronique}

Michel Baudouin, « Nozick, le liberatisme et l'utopie des dix robinsons », Labyrinthe [En ligne], 29 | 2008

(1), mis en ligne le 11 janvier 2010, consulté le 10 décembre 2020. URL : http://

journals.openedition.org/labyrinthe/3433 ; DOI : https://doi.org/10.4000/labyrinthe.3433

Propriété intellectuelle 


\section{NOZICK, LE LIBERTARISME ET L'UTOPIE DES DIX ROBINSONS}

Michel Baudouin baudouinz@free.fr

Friedrich von Hayek regrettait l'absence d'une utopie néolibérale plus séduisante que l'utopie socialiste dont il diagnostiquait le succès secret dans les politiques keynésiennes menées après-guerre aux ÉtatsUnis et dans la majeure partie de l'Europe. Maintenant que ces politiques elles-mêmes semblent être devenues des chimères gauchistes, pourquoi serait-il encore utile de faire miroiter le meilleur des mondes capitalistes possibles à l'horizon de notre imagination et de nos désirs ? L'économie libéralisée est en effet présentée par ses thuriféraires comme un fait historiquement indépassable. La chute des pays communistes prouverait qu'il n'y a jamais réellement eu d'autres voies possibles ni souhaitables. D'un autre côté, toutes nos difficultés économiques et sociales sont imputées par les mêmes personnes à un déficit de libéralisme, voire à une prégnance surnaturelle du marxisme. Libéraliser davantage est donc nécessaire pour échapper à la faillite menaçante d'économies trop peu compétitives car toujours trop étatisées, réglementées et entravées par les systèmes de solidarité sociale, de même que les sacrifices imposés pour satisfaire les nécessités économiques présentes seront à long terme récompensés. Par conséquent, le néolibéralisme est soit un fait indiscutable qui écrase toutes les utopies potentiellement concurrentes en les rejetant dans les poubelles de l'histoire, soit l'utopie que nous ne cesserons jamais de poursuivre dans un monde rétif à la rationalité économique.

Pour des raisons différentes, ses détracteurs dénoncent eux aussi une dimension utopique du néolibéralisme. Ainsi, selon Noam Chomsky, la plupart des gouvernements qui promeuvent l'économie libéralisée dans le monde et à l'intérieur de leurs frontières ne mènent pas des politiques véritablement libérales. Ce paravent idéologique 
cacherait la réalité de la guerre économique mondiale ou de la lutte consciente des classes les plus favorisées contre les moins favorisées. Les recettes économiques néolibérales seraient toujours applicables aux pays pauvres ou aux pauvres de tous les pays qu'il faut pouvoir exploiter sans être gêné par des barrières douanières ou des protections diverses, mais elles ne seraient pas valables pour les pays riches qui veulent protéger leurs marchés nationaux ou pour les riches soucieux de mutualiser les risques et de privatiser les bénéfices. Le néolibéralisme ne serait donc qu'un outil de domination pour mettre l'État et l'économie au service des puissants, aux échelles mondiale et nationale. Le néolibéralisme est alors dénoncé comme une utopie mensongère qui, réalisée, n'accoucherait sans doute pas du monde promis par ses défenseurs, mais qui, formulée, peut rallier de nombreuses personnes dont elle dessert les véritables intérêts.

Pour ses défenseurs comme pour ses détracteurs, la dimension utopique du néolibéralisme est donc constitutive des politiques économiques inféodées à cette idéologie, soit en tant que moteur, soit en tant qu'appât trompeur. Quel est donc le noyau de cette utopie? Parmi les fétiches séduisants qu'agitent les néolibéraux, la liberté individuelle occupe une place de choix. Selon la vulgate néolibérale, entraver les choix individuels par des interventions étatiques est une erreur économique et une faute politique, car celles-ci sont injustes et ne peuvent que troubler l'harmonie économique spontanée - quand elles ne nous conduisent pas tout droit au collectivisme. C'est pourquoi il peut être pertinent de chercher l'utopie néolibérale chez Robert Nozick (1938-2002), l'une des figures majeures (avec Murray Rothbard et David Friedmann) du libertarisme, une philosophie politique qui a pour principe fondamental l'inviolabilité absolue des droits individuels. Les libertariens défendent une doctrine libérale et libertaire, anarcho-capitaliste, dont certains principes, aux conséquences contrastées, ont trouvé des partisans de droite comme de gauche. En effet, si toute intervention de l'État entraîne des contraintes pour les individus et si aucun empiètement sur les droits individuels n'est justifiable, il faut tout autant condamner les politiques redistributives du Welfare State (qui impliquent de voler les uns pour donner aux autres) que les obligations militaires (qui ne sont qu'un esclavage pur et simple), que l'interdiction du commerce de la drogue (au nom de quoi empêcher quelqu'un de se droguer?), que le contrôle des flux 
migratoires aux frontières ou la moindre limite à la liberté d'expression.

On pourrait croire les libertariens opposés à tous les pans sécuritaires des politiques néolibérales récentes, au nom justement de l'utopie néolibérale elle-même, en partie caractérisée par une phobie affichée de l'État. La libéralisation des marchés du travail, du commerce et de la finance mondiale a été accompagnée de politiques pénales, policières et sociales de plus en plus contraignantes pour les catégories les plus défavorisées de la population. Aux États-Unis, les politiques conduites en direction des pauvres depuis Reagan n'ont ainsi pas diminué les interventions de l'État : celles-ci ont été simplement transférées du domaine social au domaine pénal - en même temps que les subventions étatiques ne bénéficiaient plus aux pauvres mais à des sociétés carcérales privées ${ }^{1}$. Certains diagnostiqueront ici une contradiction provisoire et accidentelle due à une libéralisation incomplète de l'économie encore entravée par l'étatisme. D'autres y verront au contraire la vérité du néolibéralisme. Ainsi, selon Foucault, la complémentarité de la liberté et de la sécurité est au cœur de la gouvernementalité néolibérale². En produisant les libertés nécessaires à leur fonctionnement, les gouvernements libéraux produisent aussi des dangers nouveaux liés à des processus non contrôlés. En contrepartie, ils doivent donc limiter cette exposition des individus au danger en étendant de façon massive les procédures de contrôle, de contrainte, de coercition. Les libertariens hurleraient peut-être contre cet étatisme tatillon, coercitif et contraire aux libertés individuelles élémentaires, mais seulement après avoir protesté et lutté de toutes leurs forces contre les impôts qui financent, entre autres choses, les aides sociales. Aux États-Unis, les conséquences extrêmes de leurs théories ont été sacrifiées aux alliances avec les conservateurs de droite : les libertariens sont assez conciliants pour ne pas s'opposer à la guerre contre la drogue ou au renforcement des contrôles frontaliers. L'abandon des conséquences libertariennes est-il fortuit et opportuniste, ou est-il inscrit au cœur de leur utopie?

L'ouvrage clé de Nozick, Anarchie, État et Utopie (1974), nous guidera pour répondre à cette question. Tout part de la question de

1. Loïc Wacquant, Punir les pauvres, Marseille, Agone, 2004.

2. Michel Foucault, Naissance de la biopolitique. Cours au Collège de France, 1978-1979, Paris, Gallimard/Seuil, 2004. 
savoir quelle place les droits individuels, considérés comme absolus et inviolables, laissent à l'État. Afin de réfuter Rothbard, pour qui le respect des droits individuels nécessite la disparition de l'État au profit d'agences de protection privées concurrentes auxquelles les individus contribuent librement, Nozick commence par établir qu'un État minimal, défini par le monopole sur la violence physique légitime, est juste et nécessaire car il n'empiète pas sur la liberté des individus protégés contre leur gré ou malgré leur pauvreté. L'argumentaire est normé par un principe fondamental : aucun sacrifice individuel n'est justifiable au nom de l'intérêt général. Le calcul qui conduit parfois, au niveau individuel, à accepter de subir un mal afin d'éviter un mal plus grand ou d'obtenir un bien, ne peut pas être étendu à l'intérêt d'une collectivité. Nozick récuse en effet l'intérêt général comme fictif et mensonger :

Il n'existe pas d'entité sociale ayant un bien qui subisse quelque sacrifice pour son propre bien. Il n'y a que des individus, des individus différents, avec leur vie individuelle propre. Utiliser l'un de ces individus pour le bénéfice d'autres, c'est l'utiliser et en faire bénéficier les autres. Rien de plus. Ce qui arrive, c'est que quelque chose lui est fait, pour le bien des autres. Parler de bien social général, c'est dissimuler cela. [...] Utiliser une personne de cette façon ne respecte pas suffisamment ni ne prend en considération le fait qu'elle est un individu séparé, que c'est la seule vie qu'elle ait. Elle ne tire aucun bénéfice marquant de son propre sacrifice, et personne n'est en droit de l'y forcer - a fortiori pas un État ou gouvernement qui revendique son allégeance (alors que les autres individus ne le font pas) et qui, par là même, se doit d'être scrupuleusement neutre face à ses citoyens ${ }^{3}$.

Sur cette base très contestable d'individualisme ontologique et moral, la nécessité et la légitimité de la formation d'un État minimal doivent être soigneusement argumentées. À partir d'un état de nature plutôt paisible, Nozick suppose que des associations volontaires d'individus se forment spontanément. Entre autres fonctions, ces associations assurent la protection de leurs membres. Mais comme ces membres n'ont pas toujours le temps de se protéger les uns les autres, et parce que la division du travail est le remède le plus rationnel à ce

3. État, Anarchie et utopie, Paris, PUF, 1988, p. 52. 
problème, Nozick en déduit que des agences privées de protection louant leurs services à des clients doivent finir par émerger. Former un État minimal consiste alors à étendre les services d'une agence privée de protection exerçant sur un territoire donné à des individus refusant ou ne pouvant pas se payer de tels services (les «indépendants»). Or, d'une part, cette extension pourrait représenter une spoliation des clients légitimes et volontaires qui paieraient la sécurité d'autres qu'eux à travers la redistribution des services de l'agence. D'autre part, l'extension pourrait constituer aussi une atteinte aux droits des individus intégrés contre leur gré à l'agence. Sachant que la solution ne doit être fondée ni sur une logique redistributive (illégitime) ni sur l'intérêt général (fictif), il reste la possibilité suivante, fondée sur un principe de compensation : si la prohibition d'actions dangereuses pour les autres est légitime, il n'en reste pas moins que la limitation de la liberté individuelle induite par la prohibition doit être compensée par quelque chose. Or des individus qui rendraient leur propre justice sur un territoire géré par une agence protectrice privée unique, seraient dangereux car ils entreraient en conflit avec les clients de celle-ci. Interdire la justice privée est donc nécessaire et légitime afin d'éviter l'insécurité qu'elle engendre, mais offrir un contrat de protection gratuit aux indépendants en compensation de cette interdiction l'est tout autant. Ainsi naît un État minimal détenteur d'un monopole sur l'usage légitime de la violence.

L'État minimal réalise l'utopie politique, le meilleur des mondes imaginables selon Nozick :

Le canevas d'utopie que nous avons décrit est équivalent à l'État minimal. [...] L'État minimal nous traite comme des individus inviolés, qui ne peuvent pas être utilisés de certaines façons par d'autres, comme moyens, outils, instruments, ou ressources. [...] Nous traitant avec respect et respectant nos droits, il nous permet individuellement ou avec ceux que nous choisissons, de choisir notre vie et de réaliser nos desseins et notre conception de nous-mêmes, dans la mesure où nous pouvons le faire, aidés par la coopération volontaire d'autres individus possédant la même dignité. Comment un État ou un groupe d'individus ose-t-il en faire plus? Ou moins ${ }^{4}$ ?

4. Ibidem, p. 405. 
C'est pourquoi Nozick tente ensuite de démontrer, contre les utilitaristes, les marxistes et Rawls, l'illégitimité d'un État que ses fonctions plus étendues forceraient à empiéter sur la liberté individuelle par des sacrifices imposés aux individus au nom de l'intérêt général fictif. L'argumentaire déployé vise surtout à réfuter l'idée qu'un État plus étendu est justifié par la nécessité de corriger les inégalités engendrées spontanément par la vie sociale ou par les rapports de force qui la structurent, en redistribuant les biens sociaux selon des normes quelconques.

Nozick distingue trois sujets de discussion à propos de la justice touchant à la propriété : l'acquisition originelle des biens, le transfert de ces biens et la réparation en cas d'injustice. En liant l'idée d'acquisition à celle du transfert des possessions d'une personne à une autre, une définition inductive, parfaite et suffisante dans un monde juste, de la justice est concevable :

a) une personne qui acquiert une possession en accord avec le principe de justice concernant l'acquisition est habilitée à cette possession;

b) une personne qui acquiert une possession en accord avec le principe de justice gouvernant les transferts, de la part de quelqu'un d'autre habilité à cette possession, est habilité à cette possession;

c) nul n'est habilité à une possession si ce n'est par application (répétée) des deux premières propositions.

Cependant, une distribution n'est pas nécessairement juste si elle naît d'une autre distribution juste grâce à des moyens légitimes : que les victimes d'un voleur aient pu lui offrir volontairement des cadeaux n'habilite pas le voleur à posséder ces gains mal acquis. Nozick défend donc une théorie «historique» de la justice, fondée sur un principe d'habilitation. La justice d'une distribution est déterminée par l'examen de l'origine historique réelle de la propriété et de l'habilitation des individus à revendiquer cette propriété. Comme nous ne vivons pas dans un monde juste, l'idée de réparation de l'injustice dans les possessions s'impose. Idéalement, un principe de réparation comprendrait une information historique sur les injustices passées et une information sur l'évolution réelle des événements nés de ces injustices - on en déduirait des estimations sur ce qui aurait dû se produire sans ces injustices afin de les réparer.

La théorie historique de la justice s'oppose aux théories où la justice d'une distribution est déterminée exclusivement par la réparti- 
tion finale des biens sociaux, indépendamment des processus individuels qui y conduisent; elle s'oppose aux théories où assurer la justice sociale consiste à réaliser et conserver un modèle prédéfini, une configuration quelconque de répartitions des biens. Or ces théories ne seraient justes que si elles avaient pour tâche de distribuer un bien qui n'a été produit par personne et sur lequel personne ne peut établir de revendications fondées. Le fait que les possessions ne tombent pas du ciel comme une manne divine, mais dépendent d'un processus de production au cours duquel des individus ont établi sur elles des droits, invalide les modèles redistributifs. La répartition des biens dans une société résulte exclusivement des nombreuses décisions individuelles.

On objectera que la justification de la redistribution découle de la coopération où tous les apports individuels à la production des biens sociaux se confondent - ce qui conduit à mettre en commun la production totale avant de la partager à nouveau selon un critère quelconque. Nozick soutient au contraire que déterminer l'apport de chacun dans la production est tout à fait vraisemblable. C'est l'exemple le plus pur du principe d'habilitation dans la possession selon un processus historique qui révèle ici la vision libertarienne de la société : si dix Robinsons, isolés sur des îles distantes, n'apprenaient leurs existences mutuelles que longtemps après avoir tout bâti chacun par leurs propres efforts, aucun d'entre eux ne pourrait réclamer avec justice une redistribution des ressources produites séparément, sous prétexte que son île était plus déserte, qu'il était moins doué, qu'il n'a pas eu de chance, etc. Chaque Robinson mérite ce qu'il obtient sans aide, par ses propres efforts et, surtout, aucun autre Robinson ne peut avoir le moindre droit de justice contre les avoirs des autres. C'est donc à partir d'une situation de non coopération sociale que Nozick justifie un principe de justice applicable à notre vie sociale. Ce n'est pas faire une grande violence au texte de penser que les dix Robinsons, c'est nous dans le monde utopique de Nozick, à l'abri d'un État prédateur désireux de nous dépouiller du fruit de nos efforts pour en faire bénéficier d'autres, moins méritants.

Redistribuer les biens est donc injuste. Pire encore : corriger les inégalités est liberticide comme le montre l'argument «Wilt Chamberlain» (nom d'un basketteur américain célèbre). En effet, si l'on part d'une situation ( $\mathrm{S} 1$ ) où tous les individus disposent d'une même somme d'argent qu'ils peuvent dépenser librement, certains d'entre 
eux épargneront, d'autres paieront les études de leurs enfants, d'autres encore iront voir jouer Wilt Chamberlain, un basketteur si talentueux et charismatique qu'il attire les foules et mérite de toucher une commission sur chaque ticket acheté. Au bout de quelque temps, S1 fera spontanément place à une nouvelle situation (S2), où l'argent sera distribué inégalement. Le basketteur est devenu plus riche que beaucoup d'autres individus. En quoi S2 est-elle injuste? En quoi ce transfert peut-il donner lieu à des revendications de justice distributive, par une tierce personne qui n'avait aucun droit de justice sur la moindre possession avant le transfert, sur une portion de ce qui a été transféré ? Aucun principe de justice distributive mis en modèle ne peut donc être appliqué bien longtemps sans des interventions permanentes et liberticides de l'État dans la vie des gens : maintenir un modèle implique en effet d'intervenir continuellement pour empêcher des transferts volontaires de ressources entre individus, ou pour ôter à certaines personnes des ressources que d'autres choisissent de leur transférer. La redistribution implique nécessairement de graves atteintes aux droits individuels. Au contraire, l'exercice des libertés individuelles bouleverse les modèles réalisés conformément à des principes finaux quelconques ou ayant une composante égalitaire forte. Ainsi, à moins qu'on ne les interdise, des actions capitalistes surgiraient dans une société socialiste et finiraient par la transformer. Un modèle de redistribution plus faible conduit au dilemme suivant : soit il est instable, soit il se satisfait du système de l'habilitation.

L'existence d'une fiscalité obligatoire utile pour redistribuer les biens sociaux est ainsi une des raisons pour lesquelles Nozick jugeait le monde injuste et sa théorie utopique. Toute solidarité qui n'est pas contractuellement acceptée par l'individu est injuste. Peu importe qu'elle ait pour but de faire fonctionner des systèmes de santé, d'éducation ou d'aides sociales : le contribuable n'est jamais qu'un Robinson spolié. Une sensibilité philosophique formée en France aura sans doute des difficultés à prendre au sérieux la robinsonnade outrancière ou l'usage d'outils philosophiques datés (comme l'état de nature). D'aucuns se demanderont si Nozick parle d'une réalité quelconque ou s'il se contente de jouer avec des concepts. Pourtant, Nozick est sans doute avec Rawls l'un des philosophes politiques américains les plus réputés et les libertariens ont été financés par des think tanks favora- 
bles au néolibéralisme. En outre, les conséquences pratiques possibles des jonglages logico-politiques libertariens ne sont pas négligeables, et l'histoire montre que même les discours grotesques suscitent parfois l'enthousiasme.

L'utopie de l'État minimal n'a qu'une fonction concrète possible : discréditer toutes les formes instituées de solidarité en dénonçant leurs effets pervers, selon une figure chère aux discours réactionnaires. L'absence d'une théorie du passage menant du monde actuel (injuste) à cette utopie le montre à double titre - d'abord parce qu'elle est inutile, ensuite parce qu'elle est destructrice pour le libertarisme. Philippe Van Parijs a déjà souligné que le point faible du libertarisme est le critère de propriété légitime ${ }^{5}$. Comment justifier la propriété d'objets extérieurs? Ces objets sont toujours le résultat de la combinaison d'un travail humain et de ressources naturelles n'appartenant à personne. Pour amorcer un processus légitime de transfert des possessions, il faut donc énoncer un principe d'appropriation originelle. La difficulté apparaît si l'on remarque qu'avant l'appropriation privée d'une ressource naturelle, tous les individus jouissaient du droit d'en user librement - si ce droit ne peut pas être considéré comme absolument inviolable, sa violation doit toutefois entraîner une compensation. C'est la raison pour laquelle Nozick adopte une «clause lockéenne», qui stipule qu'une appropriation n'est légitime que si elle n'affecte négativement le sort d'aucun autre individu. Ce «détail» n'en est pas un, car nous vivons dans un monde où les communaux ont presque totalement disparu et où subsister par ces propres moyens en vivant de chasse et de cueillette est impossible. Dans la perspective libertarienne elle-même, il faut donc admettre que les laissés-pourcompte du système ont été les victimes d'une injustice que le marché ne peut pas compenser :

«Tout ceux dont l'appropriation privée des communaux (et leur transmission ultérieure par don et par échange) a détérioré le sort ont droit au moins à une compensation les faisant accéder au niveau de bien-être auquel ils se seraient trouvés en l'absence de cette appropriation. [...] Ainsi donc, du point de vue même de la pensée libertarienne, se trouve légitimée une redistribution des revenus dont l'ampleur potentielle est considérable ${ }^{6}$.»

5. Qu'est-ce qu'une société juste?, Paris, Seuil, 1991, p. 122-125.

6. Ibidem, p. 124. 
L'assimilation de l'imposition et du vol, une idée capitale pour les libertariens, est mise à mal. Les partisans d'expropriations massives seraient sans doute surpris de recevoir le soutien de Nozick. Mais la contradiction est insoluble si l'on est favorable à une redistribution massive et obligatoire des revenus tout en réclamant à cor et à cri une réduction radicale des activités de l'État. Malgré l'usage d'un soi-disant principe historique de justice, il est donc plus commode pour Nozick d'ignorer le poids énorme de l'histoire dans la distribution réelle des possessions et dans les possibilités objectives et subjectives héritées des individus, ainsi que les rapports de force induits par ces héritages.

Or, à cause de ces impasses sur des dimensions essentielles d'une pensée politique un peu en prise sur la réalité, Nozick est obligé d'établir un postulat assez coûteux dès le départ de son entreprise : c'est l'idée que l'état de nature le plus crédible est celui de Locke et non celui de Hobbes. C'est un état de nature paisible, une anarchie tranquille où les hommes satisfont dans l'ensemble à des contraintes morales et où ils agissent, dans l'ensemble, comme ils le doivent. Chez Nozick, la philosophie morale forme l'arrière-plan et définit les frontières de la philosophie politique : ce que des individus isolés peuvent ou non se faire les uns aux autres limite ce qu'ils peuvent se faire les uns aux autres par l'intermédiaire d'un appareil d'État. La morale, qui définit les limites de la politique, prescrit le respect des droits naturels à la liberté, à la vie et à la propriété. La bonne vieille morale bourgeoise et conservatrice, chassée en apparence par le défenseur intransigeant de la liberté individuelle, n'avait donc jamais disparu. En inscrivant la liberté individuelle et la propriété à la fois dans la nature et dans la morale, Nozick castre à la source toute possibilité d'une critique historique et politique de l'état actuel du monde. Par conséquent, les libertariens ne peuvent que légitimer la libéralisation économique au nom de la liberté absolue de l'individu propriétaire et fermer les yeux sur les contraintes sociales ou pénales de plus en plus fortes exercées sur ceux qui, privés de tout accès à la propriété, finissent par perdre leur liberté soit au travail, soit en prison. 